\title{
Performance Efficient Launch Vehicle Recovery and Reuse
}

\author{
John G. Reed ${ }^{1}$ and Mohamed M. Ragab ${ }^{2}$ \\ United Launch Alliance, Centennial, Co, 80112 \\ F. McNeil Cheatwood ${ }^{3}$, Stephen J. Hughes ${ }^{4}$, J. DiNonno ${ }^{5}$, and R. Bodkin ${ }^{6}$ \\ NASA Langley Research Center, Hampton, Va, 23681 \\ Allen Lowry ${ }^{7}$ \\ Airborne Systems, Santa Ana, Ca, 92705 \\ Gregory T. Brierly ${ }^{8}$ and John W. Kelly ${ }^{9}$ \\ NASA Armstrong Flight Research Center, Edwards, CA, 93523
}

\begin{abstract}
For decades, economic reuse of launch vehicles has been an elusive goal. Recent attempts at demonstrating elements of launch vehicle recovery for reuse have invigorated a debate over the merits of different approaches. The parameter most often used to assess the cost of access to space is dollars-per-kilogram to orbit. When comparing reusable vs. expendable launch vehicles, that ratio has been shown to be most sensitive to the performance lost as a result of enabling the reusability. This paper will briefly review the historical background and results of recent attempts to recover launch vehicle assets for reuse. The business case for reuse will be reviewed, with emphasis on the performance expended to recover those assets, and the practicality of the most ambitious reuse concept, namely propulsive return to the launch site.
\end{abstract}

In 2015, United Launch Alliance (ULA) announced its Sensible, Modular, Autonomous Return Technology (SMART) reuse plan for recovery of the booster module for its new Vulcan launch vehicle. That plan employs a non-propulsive approach where atmospheric entry, descent and landing (EDL) technologies are utilized. Elements of such a system have a wide variety of applications, from recovery of launch vehicle elements in suborbital trajectories all the way to human space exploration. This paper will include an update on ULA's booster module recovery approach, which relies on Hypersonic Inflatable Aerodynamic Decelerator (HIAD) and Mid-Air Retrieval (MAR) technologies, including its concept of operations (ConOps). The HIAD design, as well as parafoil staging and MAR concepts, will be discussed. Recent HIAD development activities and near term plans including scalability, next generation materials for the inflatable structure and heat shield, and gas generator inflation systems will be provided. MAR topics will include the ConOps for recovery, helicopter selection and staging, and the state of the art of parachute recovery systems using large parafoils for space asset recovery and high altitude deployment.

The next proposed HIAD flight demonstration is called HULA (for HIAD on ULA), and will feature a $6 \mathrm{~m}$ diameter HIAD. An update for the HULA concept will be provided in this paper. As proposed, this demonstration will fly as a secondary payload on an Atlas mission.

\footnotetext{
${ }^{1}$ Principal Investigator, GN\&C Sr. Technical Fellow, 7958 S Chester St/A5000, AIAA Senior Member.

${ }^{2}$ Fmr. PI, Advanced Programs, 7670 S. Chester St, AIAA Senior Member.

${ }^{3}$ Senior Engineer for Advanced Planetary EDL Systems, Bldg 1209 Rm 120R, AIAA Associate Fellow.

${ }^{4}$ Senior Engineer for Inflatable Aeroshell Systems, Bldg 1209, Rm 130D, AIAA Senior Member.

${ }^{5}$ Senior Engineer for Mechanical Systems, Bldg 1209, Rm 130G

${ }^{6}$ Senior Engineer for Mechanical Systems, Bldg 1209, Rm 142

${ }^{7}$ Director, Inflatables and Fabric Systems, 2000 West Segerstrom, AIAA Member.

${ }^{8}$ Mission Integration Lead, Bldg. 4800, Rm 2520, AIAA Member.

${ }^{9}$ Project Manager for Space Technology Development, Bldg. 4800, Rm 1027, AIAA Senior Member.
}

American Institute of Aeronautics and Astronautics 
The Centaur upper stage provides the reentry pointing, deorbit burn, and entry vehicle spin up. The flight test will culminate with a recovery of the HIAD using MAR. HULA will provide data from a Low Earth Orbit (LEO) return aeroheating environment that enables predictive model correlation and refinement. The resultant reduction in performance uncertainties should lead to design efficiencies that are increasingly significant at larger scales. Relevance to human scale Mars EDL using a HIAD will also be presented, and the applicability of the data generated from both HULA and SMART Vulcan flights, and its value for NASA's human exploration efforts will be discussed. A summary and conclusion will follow.

\section{Nomenclature}

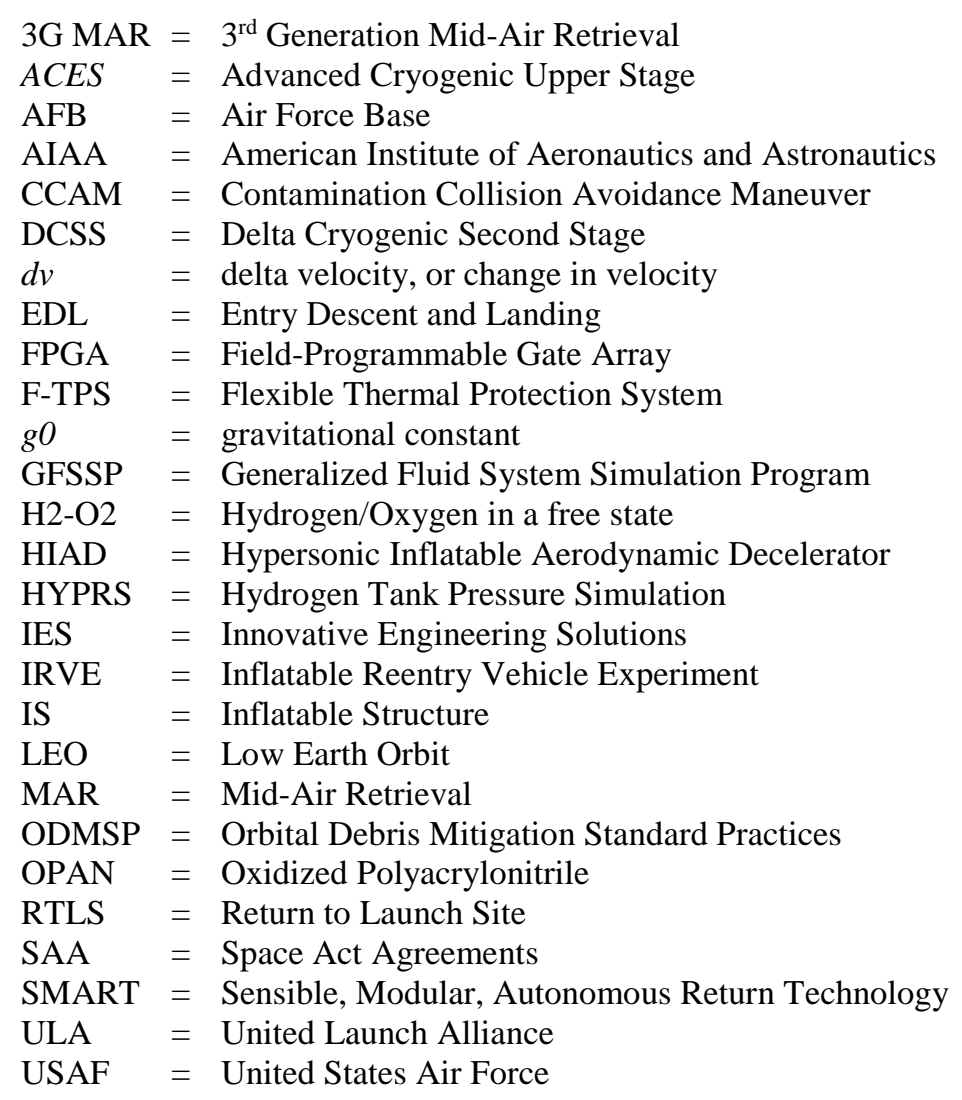




\section{Background}

$\mathrm{R}$ euse has been engrained in the vision of access to space since the early days of science fiction. While the development of the Space Shuttle matured the technologies necessary to enable development and operation of a reusable launch system, attempts to build on that heritage (Figure 1) to provide economic reuse remained an elusive goal. With recent successes in the recovery, testing and relaunch of boost vehicles, debate on the merits of various approaches to space access has been reinvigorated. The challenge of closing the business case around a reusable architecture remains key to fielding a long term viable reusable launch system. ULA has been refining its SMART Reuse concept as an approach to close the business case ${ }^{1}$.

The pace of refining the systems for fielding a commercial reusable system has clearly accelerated. After decades of operating the NASA Space Shuttle as the only even partially reusable system, followed by the years of work on the Single Stage To Orbit X33/X-34 competition, the focus and energy of companies like Scaled Composites, Blue Origin and SpaceX have been rapidly advancing the state of the art in reusable space access.

Over the course of the last 12 months we have seen huge strides. Beginning last November, Blue Origin became the first company to successfully

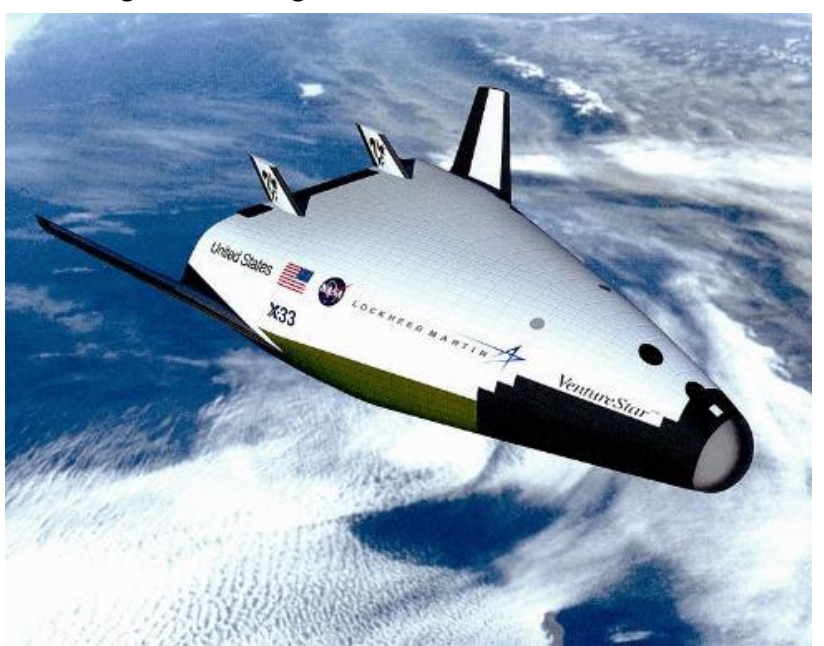

Figure 1. X-33. Credit: NASA vertically launch and land a reusable booster system. Blue Origin has repeated this process, launching and landing a total of four times with the same reusable rocket. SpaceX`s launch and return to launch site of its Falcon 9 core in December allowed them to examine and perform a static fire test on a recovered booster and further refine their design. Subequent to that milestone, they have now landed four boosters at sea (ranging from 300-680 km downrange) and followed up with a second return to launch site landing. SpaceX capped recovery of 6 of the last 11 launches with a full length hot fire of one of their recently recovered boosters. This has been a year of visually spectacular achievements in booster recovery and reuse.

\section{Business Case}

\section{Reuse}

While it is intuitive to the casual observer that it doesn't make business sense to throw away a delivery van after each delivery, the business case for space delivery is not nearly that straight forward. Clearly, the business of launch vehicles is delivery of spacecraft to orbit. As such, the typical metric from a business perspective is dollars-perkilogram to orbit. While there are other factors, like mission success probability, on-time delivery rate or time to arrival at operational orbit, these are not as directly impacted by the decision for recovery and reuse as is cost.

When evaluating reusable versus expendable launch vehicle operations the cost ratio has been found to be highly sensitive to the performance loss required to enable vehicle recovery. The previously proposed ${ }^{3} \operatorname{cost}_{\text {ratio equation (1) }}$ provides a non-dimensional definition of the reuse index comparing the impact of using the base capability of a launch system in a reusable configuration versus an expendable configuration. By manipulation of the various parameters one can quickly determine that the performance ratio is the most impactful term.

This performance ratio is the term that captures the fact that vehicle performance must be reserved to return the booster elements to earth in a manner that enables reuse. For the space shuttle, in a Trans-Atlantic Abort scenario, there was very little performance impact since the vehicle was on a ballistic path across the ocean. In that case all the energy imparted to the shuttle was dissipated by the shuttle Thermal Protection System (TPS). Even the main engines were shielded from the reentry environments by the TPS. For vertical landing of a booster this is not the case. Fuel must be reserved to remove the potential energy imparted to the booster on ascent, control the descent and minimize the impact of the reentry environments on the reusable systems.

American Institute of Aeronautics and Astronautics 


$$
I=p\left\{k\left[\frac{F}{n}+\frac{1}{n}\left(\frac{C(R H W)}{C(B)}\right)+\frac{C(R R)}{C(B)}\right]+(1-k)\right\}
$$

where:

$I=$ the reuse index

$p=$ the ratio of the performance of the expendable system to the performance of the corresponding reusable system

$k=$ the fraction of production cost of the hardware to be reused to the total cost of the expendable launch service

$F=$ a factor representing the production unit cost increase when the production rate is decreased by a factor $n$

$n=$ the number of uses

$C(R H W)=$ the reused portion of the cost to recover and reuse, such as the cost of recovery hardware that will be reused

$C(B)=$ the production cost of the hardware to be reused

$C(R R)=$ the expended portion of the cost to recover and reuse, such as recovery operation and refurbishment costs

This withheld fuel, in a non-reusable configuration, could have been used to boost additional payload mass. Given a fixed cost for launch and a conservative 30 percent allocation for simply landing at the ballistic impact point, this term is clearly significant. Even when taking into account the cost benefit from spreading the cost of key elements across multiple uses, the performance ratio is still a significant impact.

One other element in the equation that is frequently lost in the details is the fact that the hardware is not the total cost of the launch service. The normal processing operations cost for integration and launch are frequently overlooked in much of the discussion of reuse, and can further reduce the fraction of the total cost of launch impacted by reuse ( $\mathrm{k}$ in equation 1 ). This cost of taking the produced hardware from the factory door, integrating the stages and payload, and delivering the payload to the target orbit can be more than $1 / 3$ of the total cost of the launch service ${ }^{2}$.

Often the cost of infrastructure to recover and refurbish the reusable system is intuitively regarded as the most important element since this is a recurring additional cost not borne by expendable systems. While minimizing this cost is clearly of benefit, the performance ratio dominates. Simply put, to have the same $\$ / \mathrm{kg}$ to orbit, a reusable system that reduces $\mathrm{kg}$ to orbit by 30 percent to enable reusability would require a 50 percent reduction in hardware cost, including additional cost for the reusability elements, to break even.

\section{Performance Aspects of Return To Launch Site (RTLS)}

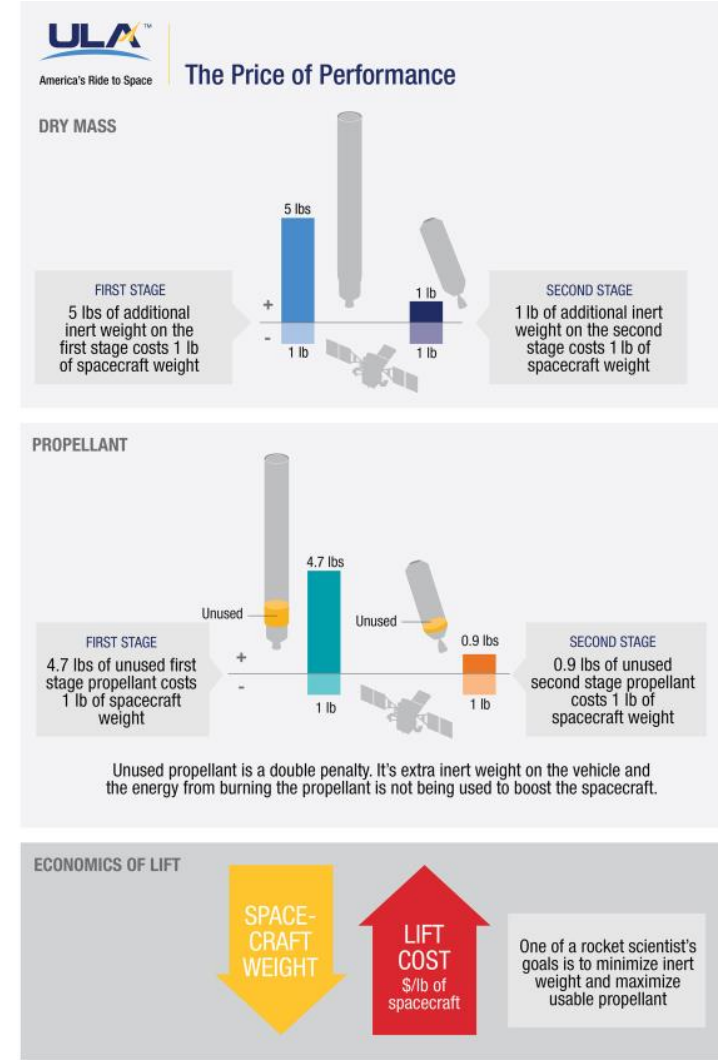

Figure 2. The cost of Performance ${ }^{4}$

Obviously the minimum recovery cost scenario is to fly the booster back to the launch site. However, the performance loss from this operation can be as much as 60 percent of the vehicle capability. It is important to operate with a common frame of reference whenever we talk percentage of capability. In this context, the fuel that is required 
to carry the stage back to its landing site could have been used to carry additional spacecraft mass to orbit. This expendable lift capability mass is the denominator in determining the percentage reduction in capability.

As shown in figure 2, while the weight relationship between upper stage and spacecraft is one-to-one, it is smaller for booster mass. This can be thought of as the lower stage and upper stage propellants work together to impart energy to the remaining mass at the end of the orbit insertion burn. The mass delivered to that desired orbit is some combination of upper stage and spacecraft, thus for every pound increase of the upper stage, there is a corresponding decrease in the mass of the spacecraft that can be delivered. The partial for upper stage fuel is slightly less favorable since the performance energy of the fuel as well as its mass must be offset. Another way to think of this is that a 1 pound reduction in fuel capacity results in a 1.1 pound reduction in lift capability.

For a booster mass increase, the partial is less than one-to-one. If the upper stage mass decreases by one pound and the booster increases by one pound, the first stage flight would be the same, but the upper stage would have a lighter payload to accelerate and would end up with a little more than a pound of excess propellant remaining. Assuming a constant upper stage mass and residuals at delivery to a target orbit, for the launch vehicle to only deliver a one pound lighter spacecraft mass to the target orbit would require a lower energy state at booster shutdown. Given a fixed propellant load on the booster and that lighter spacecraft, to end up at that lower energy state could require 5 additional pounds in the booster dry mass. The actual partial is sensitive to the specifics of booster and upper stage engine performance and the target orbit, but this is a reasonable rule of thumb for Low Earth Orbit (LEO) delivery.

The critical aspect of the performance impact for the return to launch site scenario is the amount of down range velocity delivered by the booster system. The booster has to cancel every foot per second of downrange velocity imparted to the upper stage and payload, and in fact reverse it to move the landing point from hundreds of miles down range back to the launch site. The bulk of that velocity change has to be imparted to the combined dry mass of the stage and fuel required to control the descent, thus the much larger reduction in performance to orbit for this scenario.

So, assuming the goal is to reduce the $\$ / \mathrm{kg}$ cost of access to space, the 60 percent penalty would imply that it is more economical to use the booster performance to deliver additional mass to orbit rather than reserve the fuel to safely return the booster to the landing site. With one third of the cost of access in operations, and even if the upper stage is only thirty percent of the hardware cost, this leaves half the cost of access in the booster. Thus any performance penalty of more than $50 \%$ results in an increased $\$ / \mathrm{kg}$ over using the booster in an expendable mode. The only time the return to launch site business case closes is when the optimal trajectory design requires very little downrange velocity from the booster. If there is simply the sufficient excess propellant to perform the return because of a very light payload, from a business sense either the payload has been charged a higher $\$ / \mathrm{kg}$ than necessary or the supplier has lost revenue by not "filling the truck". It is not always the case that flying the booster back to the launch site has reduced the cost of access to space.

\section{SMART Reuse Overview}

Armed with this understanding of the cost drivers, which so often results in expendable launch systems winning out over reuse, ULA set out to develop an approach that can show benefit to both spacecraft operators and shareholders. Our Sensible Modular Autonomous Return Technology (SMART) reuse concept sought to minimize the performance penalty while maximizing the dollar value of the elements returned. This effectively maximizes the reuse index in equation 1 . The concept is to minimize the performance penalty, leveraging favorable booster partials to minimize the performance impact of reuse.

The system is designed to recover the booster engines as the highest value element of the booster systems. It builds on the flight proven engine separation from the Atlas $2 \mathrm{AS}$ heritage. Rather than use vehicle performance for deceleration, the Hypersonic Inflatable Aerodynamic Decelerator (HIAD) technology is used with its flexible thermal protection system. This technology protects the Blue Origin BE-4 Vulcan engines from the thermal environments of reentry. Next, a drogue and parafoil system is deployed and the HIAD system is jettisoned. The guided parafoil allows the system to steer toward a capture zone. In the mean time, a helicopter has been proceeding into the capture zone based on the initial impact point from the Vulcan booster separation state. The two vehicles converge to

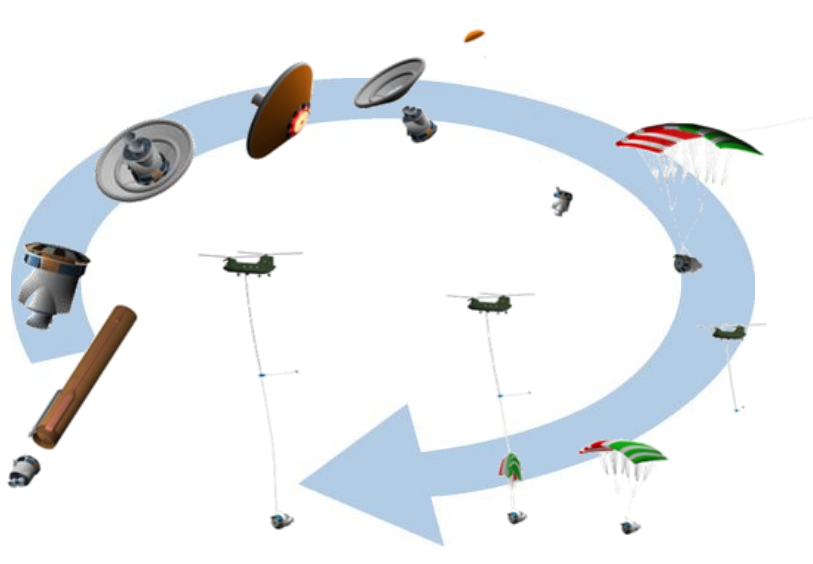

Figure 3. ULA's SMART Reuse Approach ${ }^{4}$ 
perform a Mid-Air Retrieval allowing delivery to a waiting ship.

ULA has been working with NASA Langley Research Center on maturing the HIAD concept for SMART Reuse and have honed in on a 12 meter implementation for the system. ULA has also been working with NASA Armstrong Flight Research Center and Airborne Systems to refine the MAR elements. These efforts under the NASA Space Act Agreements (SAA) have allowed development of the elements requiring further investigation with multi-program benefit. This has been a very fruitful method for collaboration as these concepts are evolved.

\section{HIAD System Design}

A Hypersonic Inflatable Aerodynamic Decelerator (HIAD) aeroshell assembly is composed of two separate assemblies: the Inflatable Structure (IS) that maintains the aeroshell shape creating the aerodynamic drag covered by the Flexible Thermal Protection System (F-TPS) that protects the IS from the aerothermal environment generated by the hypersonic entry into an atmosphere.

The IS is constructed of toroidal inflatable elements. A series of inflatable elements, each subsequent element of increasing major diameter, are stacked together to produce a cone. The inflatable elements are joined together using a network of webbing elements and are attached to the entry vehicle with additional webbing elements.
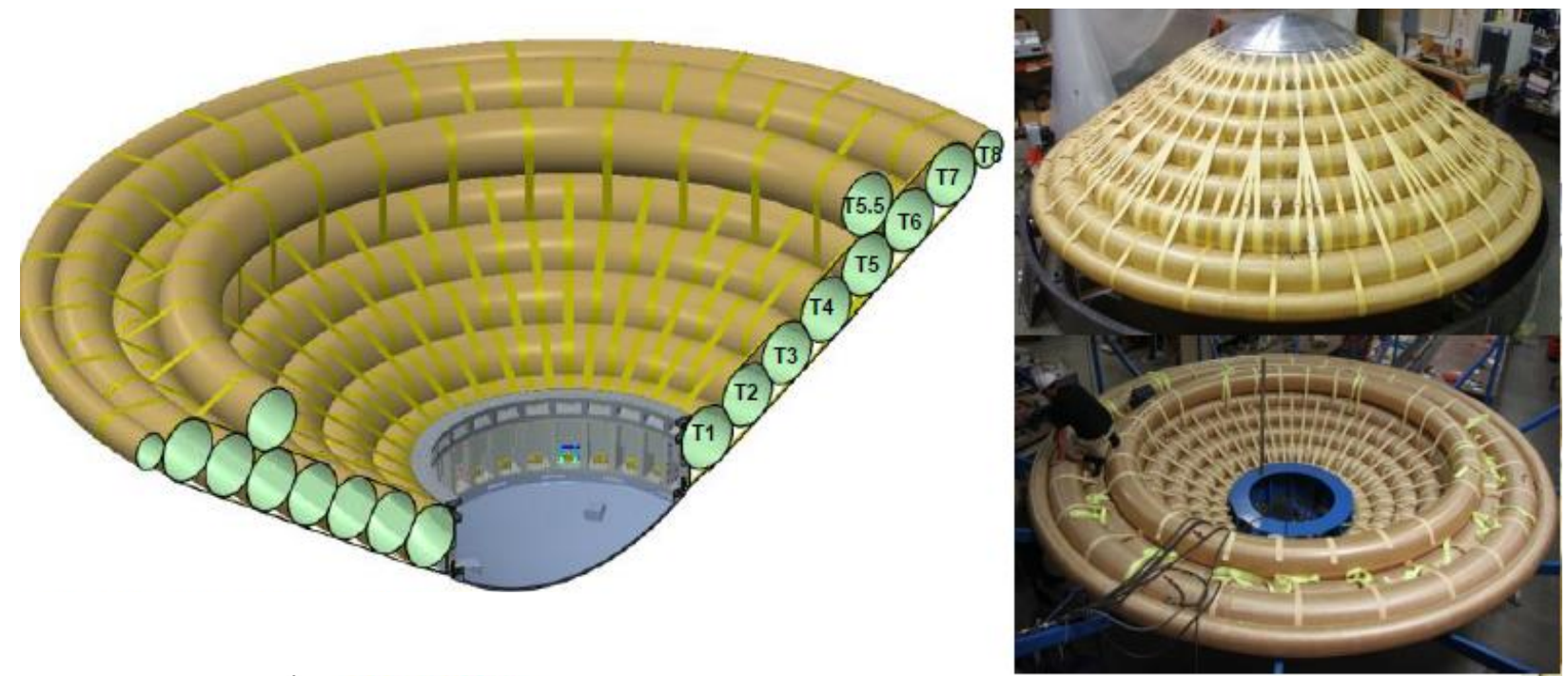

Figure 4. HIAD Inflatable Structure

The F-TPS is constructed from an outer fabric layer that protects the system from the aerodynamic shearing forces at surface temperatures generated during atmospheric entry. An insulating ply resides behind the outer fabric to limit the F-TPS back surface temperature to the IS design limit temperature. Behind the insulating ply is an impermeable layer (gas barrier) to dead head the F-TPS assembly and prevent hot gas from flowing through and reaching the IS assembly. The F-TPS is fitted over the IS. Both assemblies are attached to the entry vehicle structure, each with their own mechanical interface. 


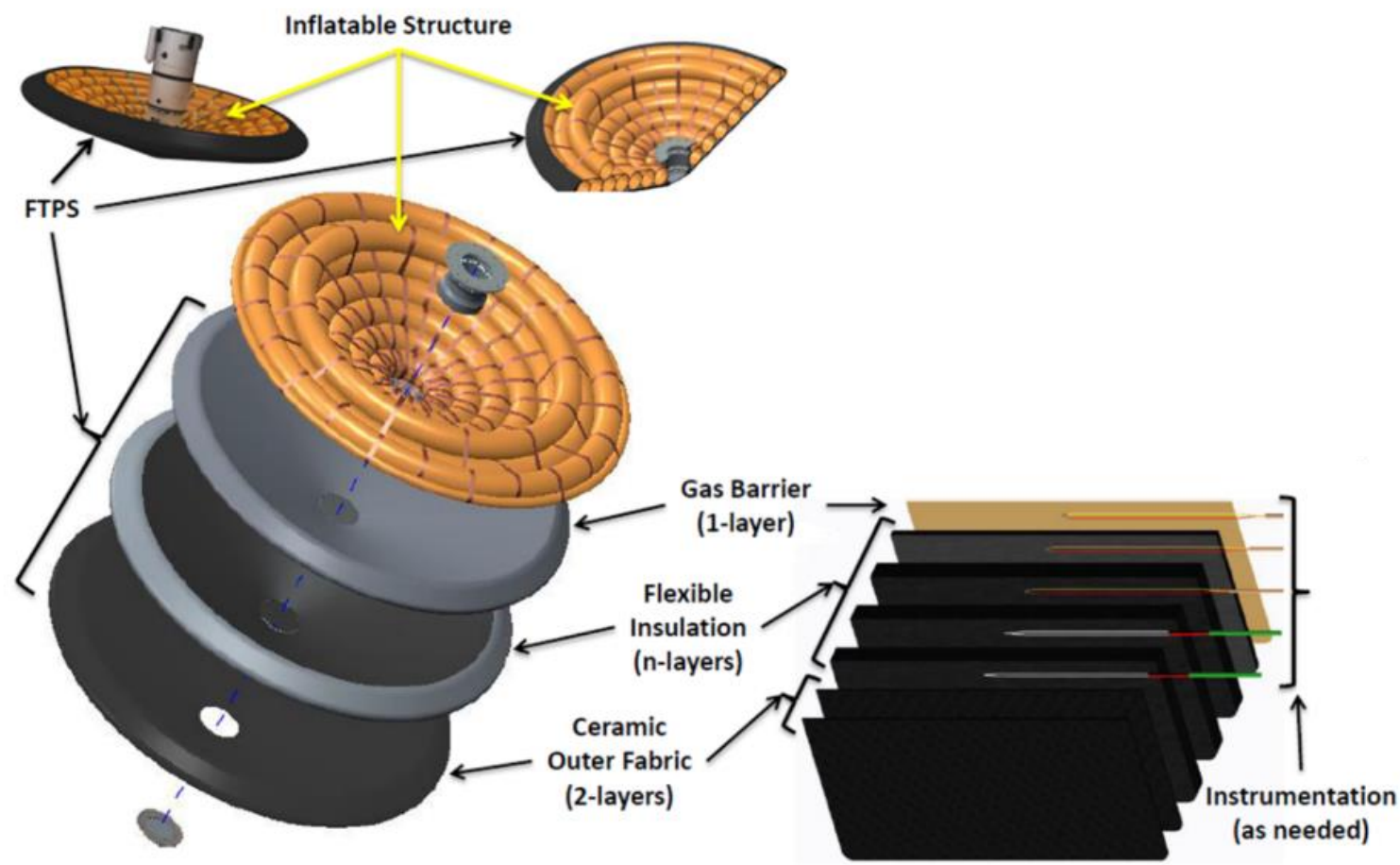

Figure 5. HIAD Flexible Thermal Protection System elements

\section{Gas Generator Inflation System}

The HIAD for the SMART Reuse will be the largest flown HIAD when it is flown. Historically IRVE-II and IRVE-3 were inflated with a nitrogen blow-down system (Figure 6). These systems were designed to utilize commercial off-the-shelf (COTS) parts to minimize system cost, and were not mass optimized. These inflation systems were robust, capable of supporting a very wide range of leak rates and inflation pressures. There were no requirements to avoid restricting access to the IRVE experiments, which is required by range safety rules for pressurized pressure vessels. ULA has indicated they do not want the SMART Reuse system to require ground support equipment to fill the system while on the pad. There are two ways to design a blow-down system. One requires a control and regulation system and the other requires a well known leak rate to design an orifice controlled blow-down system. The concern of tank leakage and many components to possibly fail has led the HIAD team to suggest gas generators for the system. Having gas generators instead of blowdown systems will allow an extremely simple system to be designed and still provide robust capabilities to support the SMART Reuse System (Figure 7). It should be noted that there is quite a bit of developmental instrumentation shown in Figure 4 that could be phased out at as the system moves from experimental to operational. This system will have a check valve and simple controller, that could be an analog or a field-programmable gate array (FPGA)

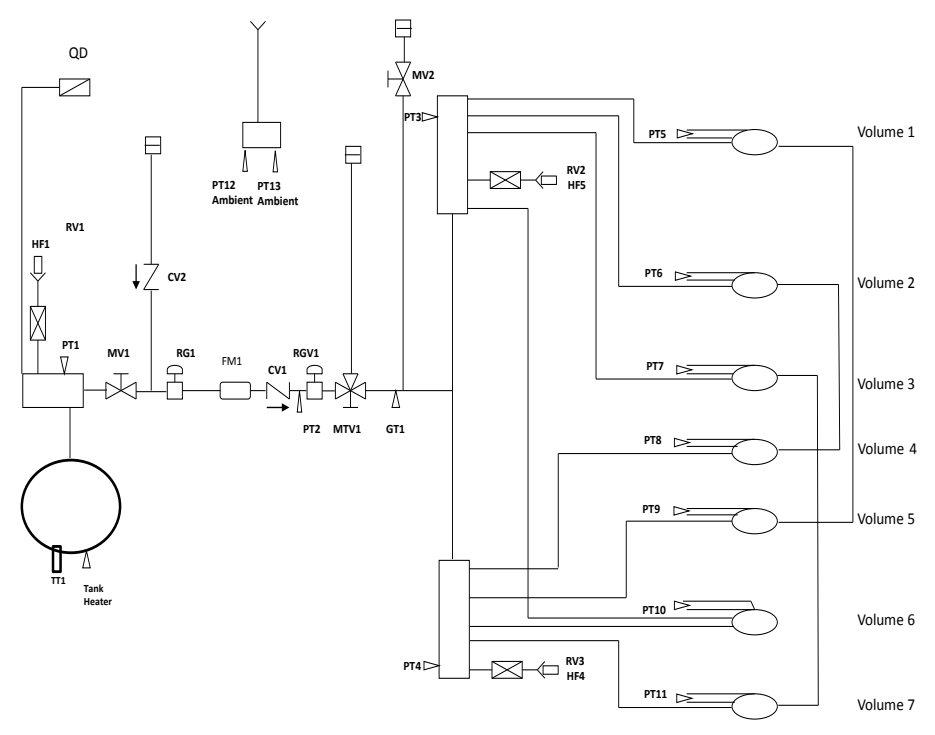

Figure 6. Blow-Down Inflation Schematic

American Institute of Aeronautics and Astronautics 
based controller, that would select which gas generator to activate.

The HIAD-2 project has started a research activity to indentify the best gas generator technology for their longterm needs. The requriments for the SMART Reuse system are similar to a human-scale Mars mission and therefore the same technologies may be utilized for both systems. The move from blow-down systems to gas generators is also opening the trade space to inflation gases other than nitrogen.

Gas generators allow several chemistries, which require smaller infrastructure on the vehicle than a blow-down system. The total system mass of the gas generator system appears to offer significant weight savings. The current trade study is honing in on three technologies for possible investement. Solid gas generators offer a technology that is very similar to car airbags or solid rocket motors. There are several chemistries that have high yields in moles of gas per mass of propellant and the output temperature can be tailored. There is a risk with the technology that when the solid propellant is enlarged to support a large scale HIAD. Cracking of the solid grain can happen, which could lead to loss of pressure containment. The second technology being researched is hybrid gas generators. These generators offer possible weight savings, but they involve a pressure vessel that uses a secondary gas to liquify a primary gas. When the system is activated, an energetic device is directed into the pressure vessel. This causes the liquid to flash evaporate and the secondary gas pressure rises from the heat, which causes the valve to open allowing the gas to move downstream. This system is still a pressure vessel with the added risk of a phase change, that may allow liquid into the infatable bladder. The final promising technology is hydride storage of gas. This system's output is at the high end of the usable temperature for the HIAD and may require additional insulation. Unfortuanately, the production of some of the more effiecient storage mediums are still on the gram scale versus the tens of kilograms that would be required for SMART Reuse. All three of these technologies could

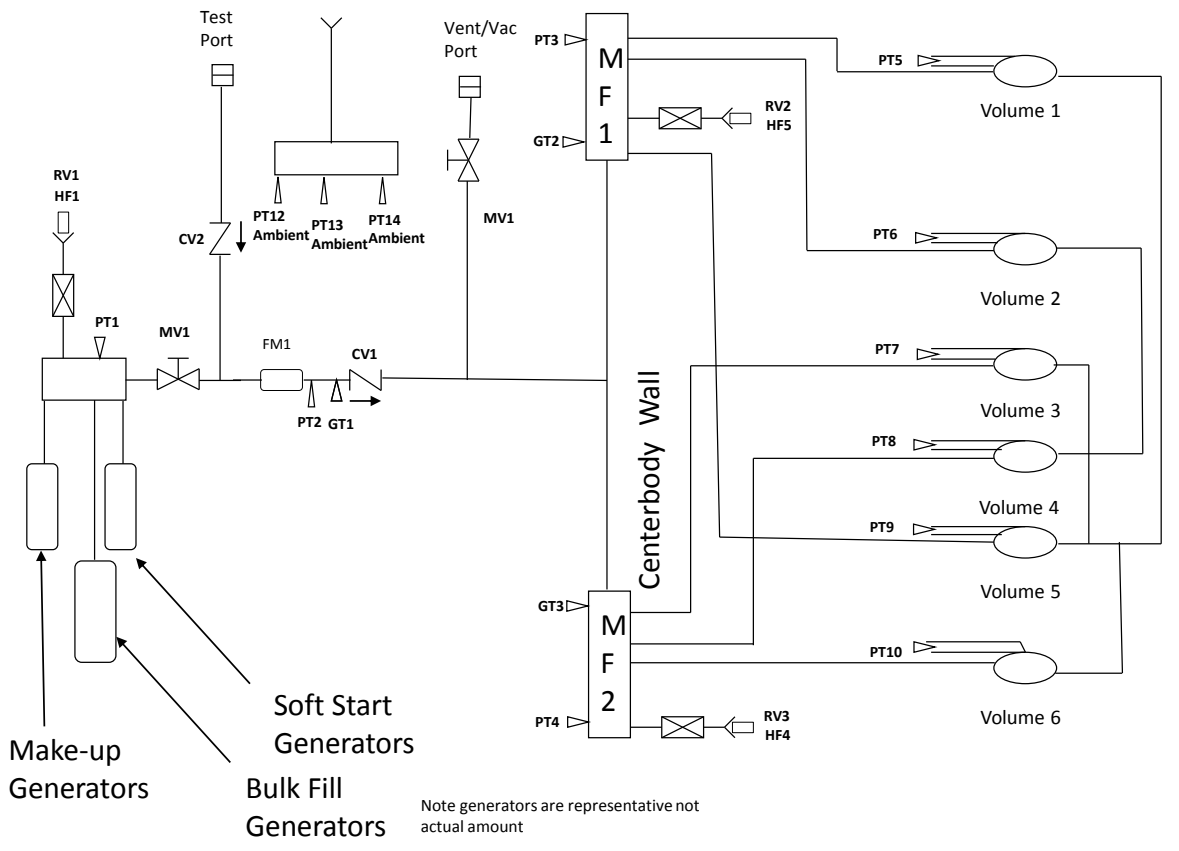

Figure 7. Gas Generator Inflation Schematic

be developed with a relatively modest investment.

\section{Further HIAD Development and Next Steps}

The modular nature of the HIAD allows for integration of material improvements without significant redevelopment or requalification. The HIAD project has been working on several material improvements for both the IS and F-TPS, along with improvements in large scale manufacturing techniques. If any developmental materials demonstrate superior capabilities they can readily be incorporated in a new hybrid layup.

\section{MAR}

NASA's Armstrong Flight Research Center commissioned a study to investigate the feasibility of the mid-air retrieval (MAR) of heavy Earth-returning space systems up to the lift capacity of the most capable commercial heavy lift helicopters. Mid-air retrieval is part of the entry, descent, and landing systems portion of NASA's 2015 technology

American Institute of Aeronautics and Astronautics 
roadmap and enables the recovery of "sensitive payloads that cannot survive a shock impact; payloads that must be kept secure; items that need to be returned to a specific location more quickly than they can be located, accessed, and transported after landing; and high-value hardware that can be reused with minimal refurbishment, to save costs". The method studied represents the 3rd Generation MAR (3G MAR) and has significant advantages over other air retrieval methods. With 3G MAR, the pickup helicopter need not be modified or re-classified as experimental as the pickup procedure conforms to Code of Federal Regulation (CFR) 14 Part 133 entitled "Rotorcraft External-Load Operations".

Prior to the completion of the study, the 3G MAR method of recovery was successfully demonstrated on an 1,100 pound test article at Edwards AFB in July 2016. This successful demonstration validates the 3G MAR system approach and provides the basis to study a more capable system.

\section{Study Scope and Methodology}

The study methodology was designed to determine the commercial viability and applicability of $3 \mathrm{G}$ MAR. Three reference missions provided a context for the examination of three mission elements that can enable efficient and reliable MAR: 1) the aero-mechanical system; 2) the concept of operations (CONOPS); and 3) guidance, navigation, and control (GN\&C) and system automation.

\section{Reference Missions}

The three reference missions represented a range of mission types and payload weights. They also represented potential near-term applications of the technology.

The Gondola for High Altitude Planetary Science (GHAPS) reference mission focused on a land-based capture of a 3,400 pound balloon-borne observatory. The HULA mission, or Hypersonic Inflatable Aerodynamic Decelerator (HIAD) on a United Launch Alliance (ULA) vehicle, focused on the recovery of the 3,750 pound HIAD test article at sea. The final reference mission focused on the recovery of the 21,000 pound first stage engines of the ULA Vulcan next generation launch system.

\section{Aero-mechanical System Study}

The aero-mechanical system study developed a conceptual design for a scalable system that can perform 3G MAR across the broadest range of weights up to the maximum lift capacity of the largest heavy-lift helicopter. Airborne Systems of Santa Ana, California was contracted to perform this portion of the study. After refining requirements and a preliminary loads analysis, a trade study of the load train elements identified the need for parafoils that can open at high altitudes (above 25,000 feet) and robust load limiting devices. The study produced a technology roadmap for the both the parafoils and load limiting devices and a conceptual design for 5,000 and 20,000 pound grapples.

\section{CONOPS Study}

The three reference missions provided a context for the development of concepts of operation and cost estimates for each mission. Erickson Aviation of Portland, Oregon was contracted to perform this portion of the study. The study identified commercial aircraft, logistics, and command and control frameworks appropriate for each mission. Land based operations are straightforward but recovery of targets at sea present some operational and rendezvous concerns. Although 3G MAR is feasible for daytime, fairweather operations with appropriate guidance to the target, more sophisticated rendezvous technology and procedures will help ensure success.

\section{GN\&C and Autonomy}

The study examined systems and techniques that would enable reliable payload/helicopter rendezvous and capture. The Charles Stark Draper Laboratory of Cambridge, Massachussets was contracted to perform this portion of the study. The study produced development roadmaps for GN\&C and autonomous systems for the parafoil, helicopter, and grapple. An aerial guidance unit on the parafoil might include a global positioning system (GPS), or an automatic dependent surveillance broadcast (ADS-B) device. Various options for parafoil steering were examined including single-line and double-line control, as well as in-canopy servos. Avionics on board the helicopter could be used to predict parafoil deployment, predict the intercept point, and provide route data to the intercept point. Although the 3G MAR baseline calls for remote maneuvering and operation of the grapple from the helicopter, avionics on the grapple could assist in the automated engagement and capture of the parafoil pickup line. The study also produced a development roadmap for mission planning tools to better predict landing ellipses and flight path planning.

\section{Conclusions}

A successful demonstration of the mid-air retrieval MAR of a 1,100 pound target provided the basis for development of 5,000 and 20,000 pound systems. Loads up to 20,000 pounds are feasible and could leverage mature, 
existing technologies. However, the load limiting device has the lowest technology readiness level (TRL) requiring some development and helicopter operations from a sea going barge present some operational and safety concerns.

\section{HULA}

\section{Context}

Hypersonic Inflatable Aerodynamic Decelerator (HIAD) on United Launch Alliance (ULA), referred to as HULA, is the proposed next flight experiment in HIAD technology development. The concept is a roughly $1700 \mathrm{~kg}$ reentry vehicle with a $6 \mathrm{~m}$ diameter aeroshell on a ballistic entry into Earth atmosphere from low Earth orbit (LEO). The 6m scale is about as large as is achievable within mass and volume constraints while targeting an appropriate heat pulse. ${ }^{5}$ The test vehicle would be delivered to its reentry trajectory as a secondary payload on a ULA Atlas V launch vehicle. ${ }^{6}$ This partnership provides a relatively low cost and near term orbital reentry flight experiment that utilizes available ULA launch vehicle performance and demonstrates Mid-Air Retrieval at a larger scale.

The largest scale and highest energy HIAD flight to date was the successful Inflatable Reentry Vehicle Experiment 3 (IRVE-3). The steep flight path angle trajectory for IRVE-3 was designed to maximize the peak heat flux available from the three stage sounding rocket for gathering flight performance data of the HIAD Flexible Thermal Protection System (F-TPS) and Inflatable Structure (IS) on a relatively low-cost experiment. The $280 \mathrm{~kg}$ IRVE-3 reentry vehicle inflated the HIAD from less than $0.5 \mathrm{~m}$ diameter when stowed to $3 \mathrm{~m}$ diameter when deployed, and demonstrated stability through hypersonic, supersonic, and subsonic atmospheric flight, undergoing $20 \mathrm{~g}$ 's deceleration to achieve about $15 \mathrm{~W} / \mathrm{cm}^{\wedge} 2$ peak heat flux. ${ }^{7}$ The ULA launch vehicle asset recovery, along with other HIAD mission infusion concepts, will be much more energetic and will involve an aeroshell on the order of $12 \mathrm{~m}$ diameter. To this end, the HIAD team at NASA and its industry partners are working on scaling up and ground testing the IS and F-TPS technology for manufacturing, handling, packing, and performance. An increased scale, high energy flight test is the next logical step in HIAD development.

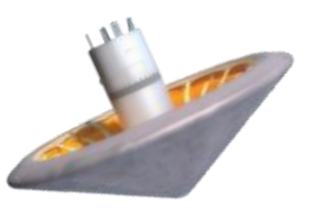

IRVE-3

3 meter diameter $60^{\circ}$ half-cone angle

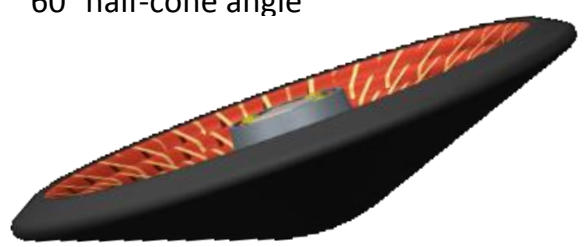

HULA

6 meter diameter $70^{\circ}$ half-cone angle

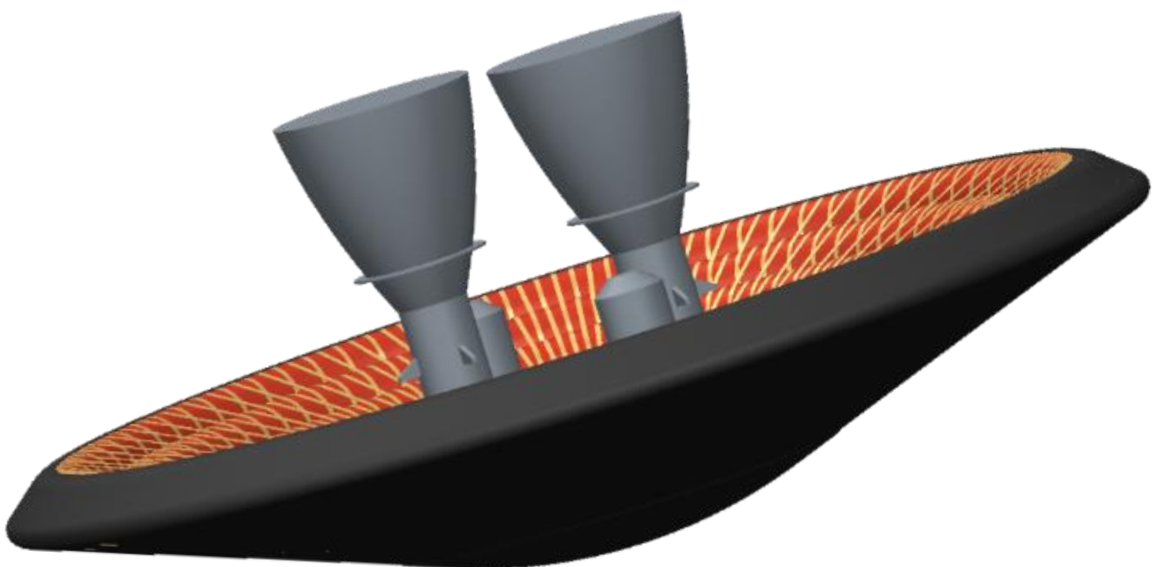

Vulcan Engine Recovery

12 meter diameter

$70^{\circ}$ half-cone angle

Figure 8. Scale comparison among IRVE-3, HULA, and Vulcan Engine Recovery

\section{Design Update}

The original HULA design concept architecture is described in the International Planetary Probe Workshop (IPPW) 13 paper: HIAD on ULA (HULA) Orbital Reentry Flight Experiment Concept. This design was based on the volume available for a particular candidate primary mission configuration, in which a HULA reentry vehicle was the only secondary payload and served as an adapter between the Centaur second stage and the primary payload. The major internal components were conservatively sized and incorporated into the three-dimensional CAD concept model, showing ample volume available for the internal systems, even as they were packaged purposefully in a way 
that did not try to minimize their usage of space. ${ }^{5}$ More recently, NASA and ULA have been collaborating to adapt the HULA configuration to a broader range of secondary payload launch opportunities. To this end, the HULA secondary payload height has been reduced and its interfaces have been updated to accommodate a CubeSat secondary payload deployment system, named the NanoRacks Mega-Deployer (NRMD), along with an EELV (Evolved Expendable Launch Vehicle) Secondary Payload Adapter (ESPA) ring, between the HULA vehicle and the primary payload.

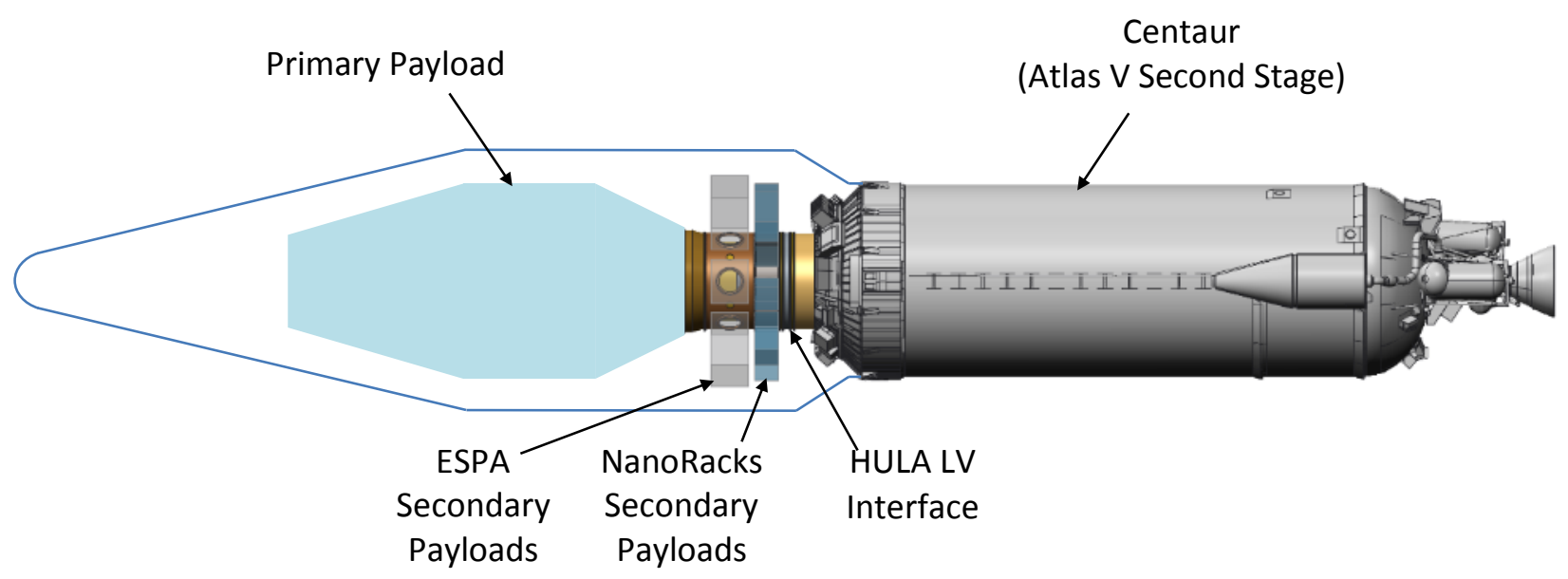

Figure 9. Atlas V Second Stage and Payload Launch Configuration

The HULA center body interface is integral to the payload adapter structure and utilizes a $1575 \mathrm{~mm}$ (62inch) RUAG ESPA Separation System interface on both the fore and aft sides. This makes the HULA stowed vehicle interface "universal" for a variety of missions, and standard ULA payload adapters can be utilized with or without additional secondary payloads. The HIAD aeroshell is packed and stowed forward of the HULA vehicle nose. The stack configuration follows, starting from the aft end of the entire payload adapter stack and moving forward.

The Centaur Forward Adapter fastens to a C-13 Launch Vehicle Adapter (13inch height), which is a cylindrical monocoque structure with the ULA Standard Interface Plane (SIP) containing a $1575 \mathrm{~mm}, 121$ hole bolt circle ${ }^{8}$ on the aft flange and an equally spaced $1575 \mathrm{~mm}, 120$ hole bolt circle on the forward flange. The C-13 adapter fastens to the clamp band retention side of a RUAG $1575 \mathrm{~mm}$ ESPA Separation System. The release side of the RUAG Separation System is included in the aft side of the HULA center body interface. The HULA center body interface aft side is mirrored on the forward side, which is fastened to the release side of a second RUAG $1575 \mathrm{~mm}$ ESPA Separation System. The clamp band retention side of the second RUAG Separation System is fastened to the aft side of the NRMD secondary payload deployment system. The forward side of the NRMD is fastened directly to the aft side of an ESPA ring (32inch height), which also utilizes the $1575 \mathrm{~mm}$, equally spaced 120 hole bolt circle interface at both ends. The forward side of the ESPA ring connects to an Atlas V Type D1666 Payload Adapter, which transitions from the $1575 \mathrm{~mm}$ diameter bolt circle to a payload separation system on a $1666 \mathrm{~mm}$ diameter bolt circle. ${ }^{8}$ The $1666 \mathrm{~mm}$ diameter separation system connects to the primary payload.

American Institute of Aeronautics and Astronautics 


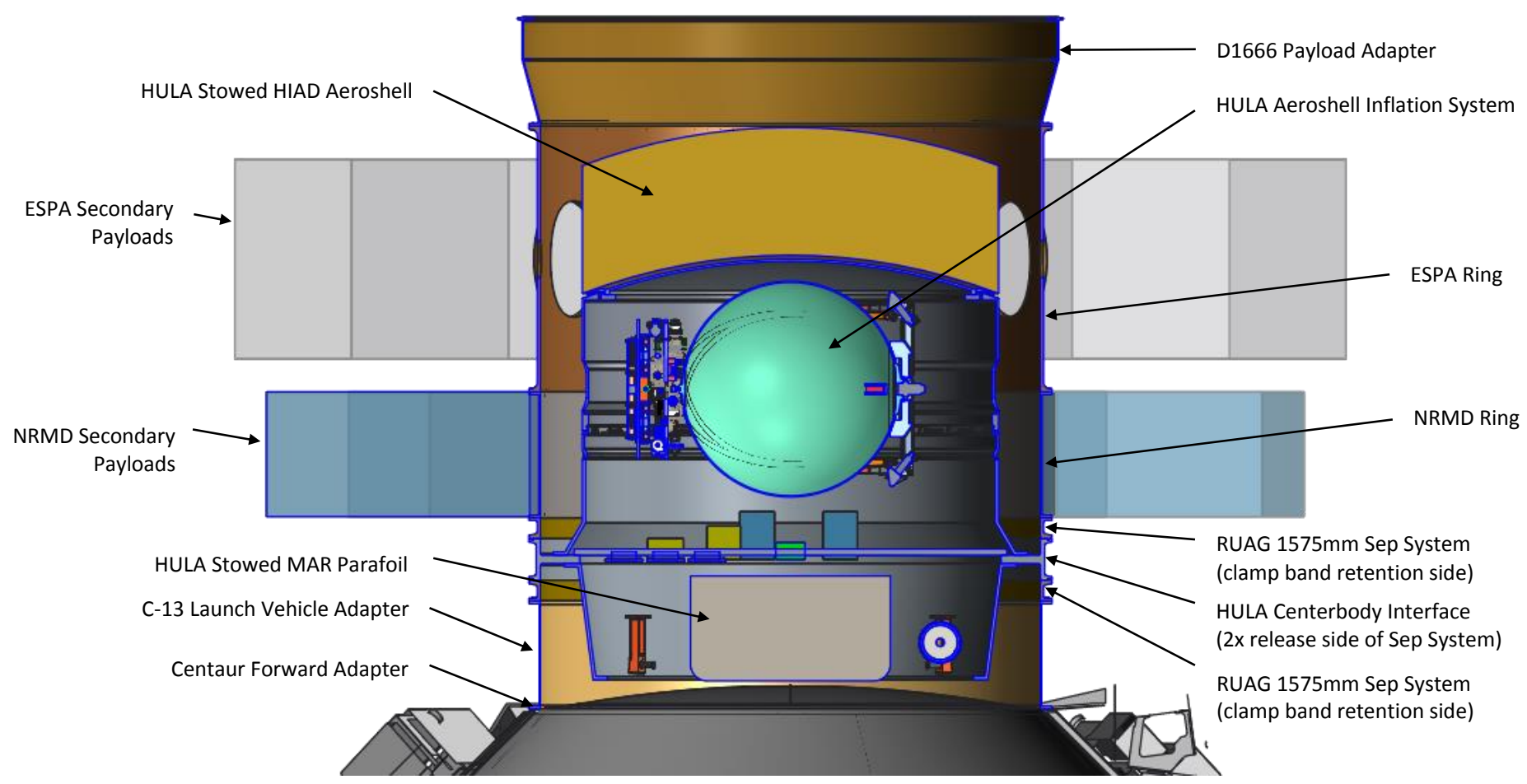

Figure 10. Payload adapter stack cross section

The HULA test vehicle concept is designed to fit within the available diameter and height of this payload adapter stack. Internal systems from the original concept were then rearranged to show that they will fit within the reduced volume of the vehicle center body.

\section{Con-ops Summary}

The HULA secondary payload is designed to do no harm to the primary payload and additional secondary payloads, and it will launch unpowered. After the primary payload, ESPA secondary payloads, and NRMD secondary payloads have been deployed and the associated Contamination and Collision Avoidance Maneuvers (CCAM) completed, the HULA test vehicle will power on. The Centaur will perform a deorbit burn to put HULA on its designed reentry trajectory. The RUAG Separation System on the forward side of the HULA centerbody interface will release the Payload Adapter-ESPA-NRMD stack. Clean separation of this adapter stack over the length of the HULA test vehicle will be assured by using a long stroke actuation system. Once clear, the aeroshell will be deployed and inflated. The Centaur will then spin up the inflated HULA test vehicle, perform a final attitude trim, and release the vehicle using the RUAG Separation System on the aft side of the centerbody interface. As the Centaur performs a divert maneuver, the HULA test vehicle will continue on its spin-stabilized ballistic reentry trajectory for the experiment. After the reentry experiment, the test vehicle will jettison a back-up data recorder and then deploy a parafoil for Mid-Air Retrieval.

American Institute of Aeronautics and Astronautics 


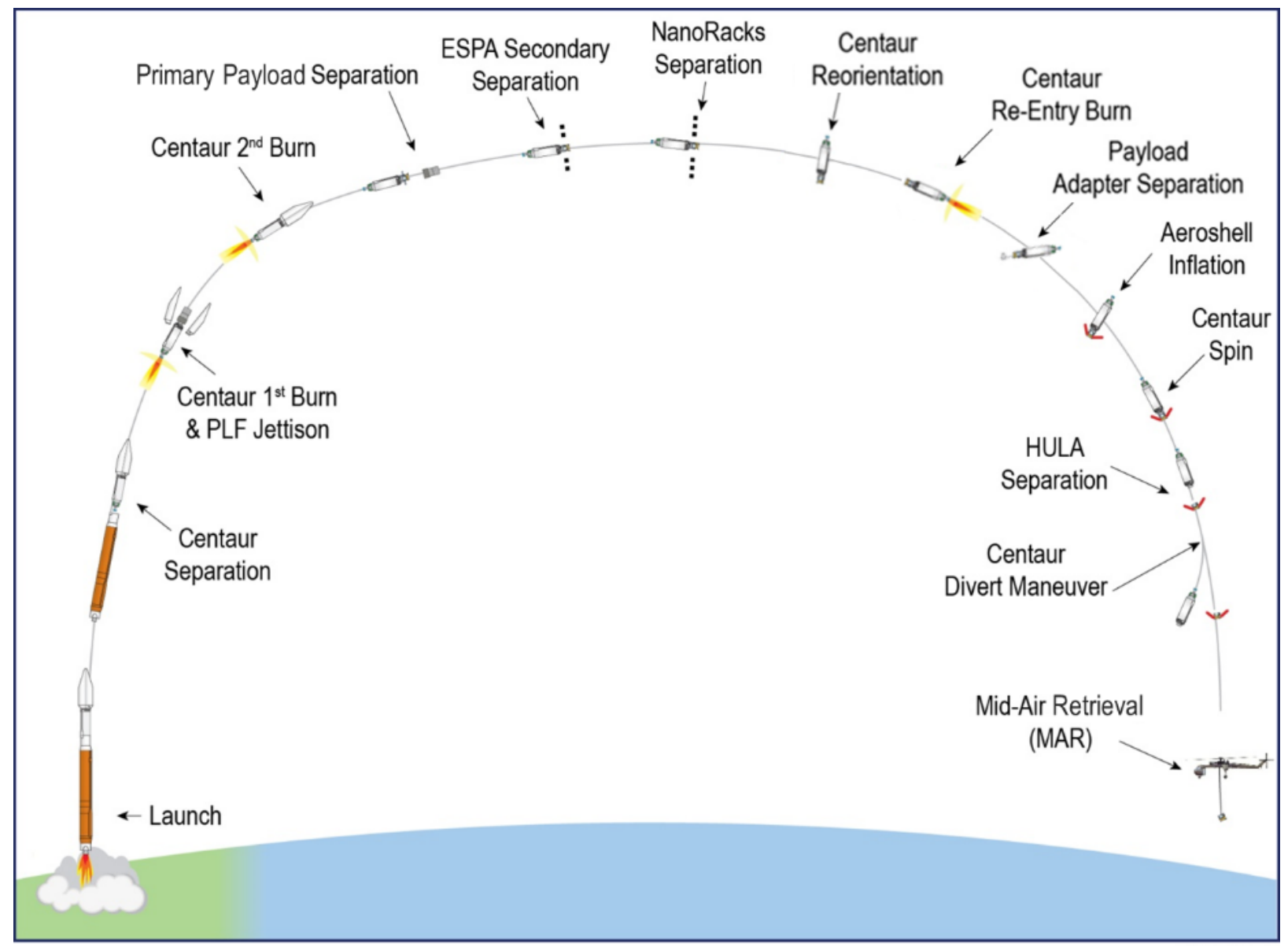

Figure 11. Concept of operations diagram

\section{HIAD Data Relevance}

Significant F-TPS design margins are required for mission applications due to uncertainties in modeling of the aeroheating environment and the associated aerothermal response, along with the inability to replicate temporally combined flight environments on the ground. HIAD technology development includes physics-based model development for the F-TPS and IS. With increased confidence in these predictive models, and with the modular material approach to HIAD, customization of F-TPS layups and IS configurations can be more accurately sized and more efficiently trade studied for hypersonic mission applications. The HULA flight experiment will provide thermal and structural response data at these design relevant conditions, which will provide increased confidence in aerodynamic, aeroheating, thermal response, and structural modeling. The flight data will also help to assess relevant ground testing, which can then further improve the analytical models. At larger diameters (large surface area), small changes in system specifications, such as F-TPS insulator thickness, may affect appreciable changes in mass and stowed volume, so fine tuning the margins will impact design and packaging requirements on larger scale missions.

American Institute of Aeronautics and Astronautics 


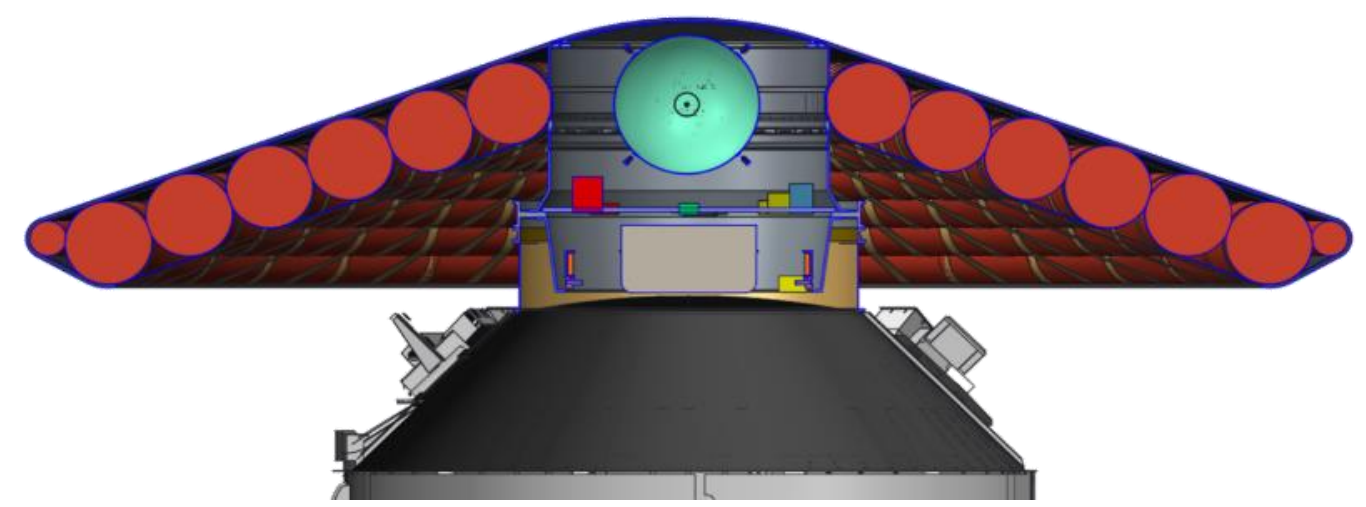

Figure 12. HULA with HIAD aeroshell inflated

\section{Data Summary}

The $6 \mathrm{~m}$ diameter HULA experiment will demonstrate the largest blunt body reentry ever flown. It will also demonstrate the largest scale Mid-Air Retrieval (MAR) operation ever performed, in both mass and drag area. The orbital reentry energy will stimulate the F-TPS materials through its thickness to provide surface and in depth thermal measurements that can refine predictive models. The $6 \mathrm{~m}$ scale provides sufficient running length to develop augmented heating due to turbulence, which can be evaluated from trajectory reconstruction, environment measurements, and thermal response measurements. Video data will provide edge deflections in flight to correlate to the structural model and ground testing. Infrared imagery will be recorded on the backside of the aeroshell, providing thermal response and aeroheating environment indications in this area of high uncertainty. The Mid-Air Recovery enables evaluation of a nearly pristine post-entry aeroshell, so the physical condition of the materials can be examined and evaluated against the recorded data, while providing additional qualitative information that flight instrumentation cannot provide. These groundbreaking demonstrations and data support the development of HIAD and MAR while feeding forward to the ULA launch vehicle asset recovery.

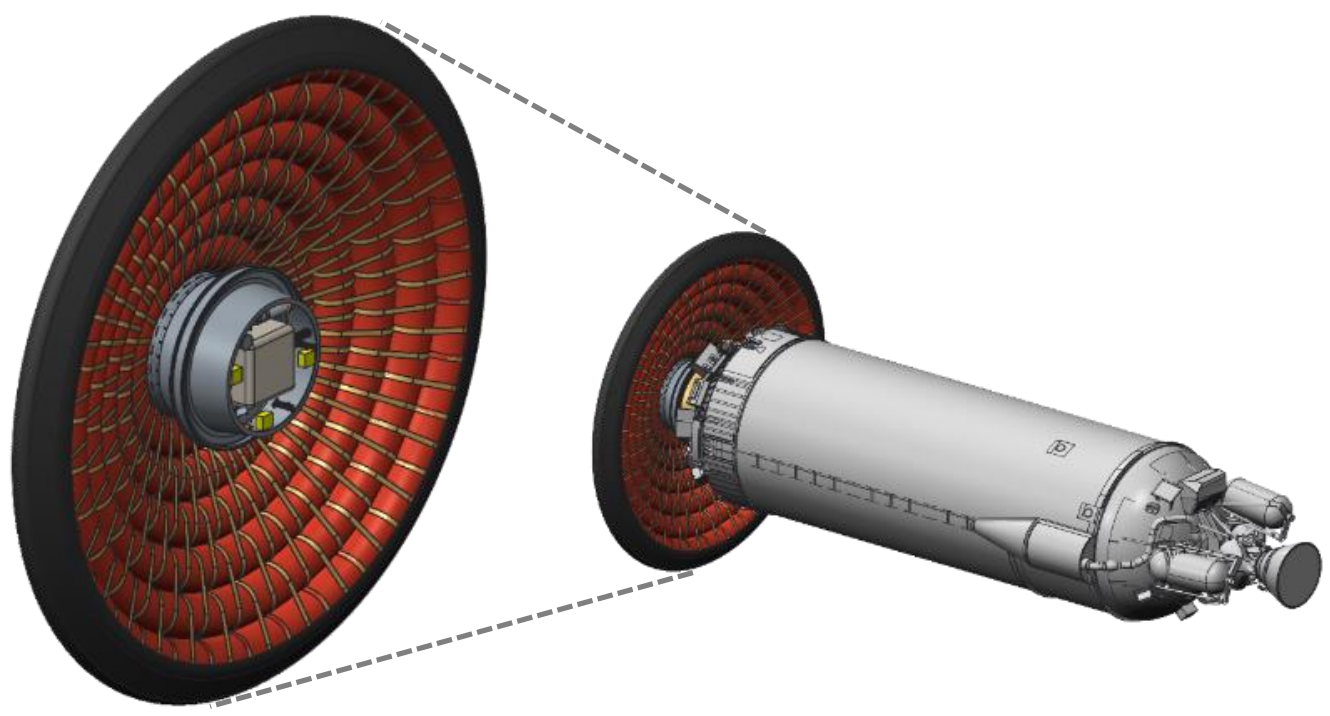

Figure 13. HULA Separated from Centaur

\section{Other Applications}

The flight test of HULA and development of a SMART HIAD system provide incremental steps on the path to planetary recovery systems. The synergies between these systems allow modest incremental expansion of the current technology base demonstrated with the IRVE flights. The system also has extensibility for inexpensive recovery of

American Institute of Aeronautics and Astronautics 
materials and products of a CIS-Lunar economy. All of these applications require deceleration from orbital speeds in order to deliver goods and components to developments on Mars or consumers on Earth. The flight regimes for these applications provide sufficient similarities to enable opportunities for common element development and production.

\section{Summary and Conclusion}

In the efforts to reduce the cost of space access the vision of a fully reusable system is not the best way forward with current technologies. The loss of performance required to return a vehicle to the launch site under propulsion erodes the systems lift capability. Given the burgeoning market for secondary mission access to space a better approach to minimize the $\$ / \mathrm{kg}$ metric for access is to minimize the performance loss for recovery.

ULA and NASA have been refining the concept for SMART Reuse which enables a minimum performance impact while recovering the highest value element of the booster, its engines. Conceptual design studies have found that a 12 meter HIAD can shield the engines with high crossover value to other applications. Preliminary cost estimates of $3 \mathrm{G}$ MAR operations for the three reference missions indicate that MAR is a small fraction of mission cost, with the reuse of the target representing a potentially significant return on investment. In total, the SMART concept has been shown to be viable with a reduction in access cost achieved after a single reuse ${ }^{4}$.

\section{References}

${ }^{1}$ Ragab, Mohamed M. Cheatwood, F. McNeil, Hughes, Stephen J. Lowry, Allen, "Launch Vehicle Recovery and Reuse," Presentation to the AIAA Infotech@ Aerospace Conference Plenary, 21 August 2013.

${ }^{2}$ Bruno, S. T. "Tory", "Continuing to get questions about the cost math from my Congressional testimony. So, here's an info graphic" Twitter, URL: https://twitter.com/torybruno/status/584032353787326464 [tweeted 3 April 2015].

${ }^{3}$ Sowers, G. F., "Reuse business case," NASASpaceFlight.com Forum, URL: http://forum.nasaspaceflight.com/index.php?topic=37390.0 [posted 23 April 2015].

${ }^{4}$ Sowers, G. F., "Analysis of SMART (engine) reuse versus stage reuse," Twitter, URL: https://twitter.com/george_sowers/status/594142397744820225 [tweeted 1 May 2015].

${ }_{5}^{5}$ DiNonno, J.M., F. M. Cheatwood, S. J. Hughes, M. M. Ragab, R. A. Dillman, R. J. Bodkin, C. H. Zumwalt, R. K. Johnson, "HIAD on ULA (HULA) Orbital Reentry Flight Experiment Concept," IPPW-13, June 2016.

${ }^{6}$ Ragab M. M., Cheatwood F. M., Hughes, S.J., Lowry, A. "Launch Vehicle Recovery and Reuse,” AIAA 2015-4490.

${ }^{7}$ Dillman, R.A., DiNonno, J.M., Bodkin, R.J., Gsell, V., Miller, N.A., Olds, A.D., Bruce, W.E., "Flight Performance of the Inflatable Reentry Vehicle Experiment 3,” IPPW-10, June 2013.

${ }^{8}$ United Launch Alliance, “Atlas V Launch Services User’s Guide,” Revision 11, March 2010.

American Institute of Aeronautics and Astronautics 